\title{
How to Prove the Riemann Hypothesis
}

\section{Fayez Fok Al Adeh*}

President of the Syrian Cosmological Society, P.O.Box:13187, Damascus, Syria

\begin{abstract}
The aim of this paper is to prove the celebrated Riemann Hypothesis. I have already discovered a simple proof of the Riemann Hypothesis. The hypothesis states that the nontrivial zeros of the Riemann zeta function have real part equal to 0.5 . I assume that any such zero is $s=a+b i$. I use integral calculus in the first part of the proof. In the second part I employ variational calculus. Through equations (50) to (59) I consider (a) as a fixed exponent, and verify that $a=0.5$. From equation $(60)$ onward I view $(a)$ as a parameter $(a<0.5)$ and arrive at a contradiction. At the end of the proof (from equation (73)) and through the assumption that (a) is a parameter, I verify again that $a=0.5$.
\end{abstract}

Keywords: Definite integral; Indefinite integral; Variational calculus

\section{Introduction}

The Riemann zeta function is the function of the complex variable $\mathrm{s}=\mathrm{a}+\mathrm{bi}(\mathrm{i}=\sqrt{-1})$, defined in the half plane $a>1$ by the absolute convergent series.

$$
\zeta(\mathrm{s})=\sum_{1}^{\infty} \frac{1}{\mathrm{n}}
$$

and in the whole complex plane by analytic continuation.

The function $\zeta(S)$ has zeros at the negative even integers $-2,-4$, $\ldots$ and one refers to them as the trivial zeros. The Riemann hypothesis states that the nontrivial zeros of $\zeta(S)$ have real part equal to 0.5 [1].

\section{Proof of the Hypothesis}

We begin with the equation,

$\zeta(S)=0$

And with,

$s=a+b i$

$\zeta(a+b i)=0$

It is known that the nontrivial zeros of $\zeta(S)$ are all complex. Their real parts lie between zero and one.

If $0<a<1$ then,

$$
\zeta(s)=\mathrm{s} \int_{0}^{\infty} \frac{[x]-x}{x^{\mathrm{s}+1}} \mathrm{dx} \quad(0<a<1)
$$

$[x]$ is the integer function.

Hence,

$\int_{0}^{\infty} \frac{[x]-x}{x^{s+1}} \mathrm{dx}=0$

Therefore,

$$
\begin{aligned}
& \int_{0}^{\infty}([x]-x) x^{-1-a-b i} d x=0 \\
& \int_{0}^{\infty}([x]-x) x^{-1-a} x^{-b i} d x=0 \\
& \int^{\infty} x^{-}([x]-x)(\cos (b \log x)-i \sin (b \log x)) d x=0
\end{aligned}
$$

Separating the real and imaginary parts we get,

$$
\begin{aligned}
& \int_{0}^{\infty} x^{-1-a}([x]-x) \cos (b \log x) d x=0 \\
& \int_{0}^{\infty} x^{-1-a}([x]-x) \sin (b \log x) d x=0
\end{aligned}
$$

According to the functional equation, if $\zeta(S)=0$ then $\zeta(1-S)=0$. Hence we get besides equation (11)

$$
\int_{0}^{\infty} y^{-1-a}([y]-y) \sin (b \log y) d y=0
$$

In equation (11) replace the dummy variable $\mathrm{x}$ by the dummy variable $\mathrm{y}$,

$$
\int_{0}^{\infty} y^{-1-a}([y]-y) \sin (b \log y) d y=0
$$

We form the product of the integrals (12) and (13).This is justified by the fact that both integrals (12) and (13) are absolutely convergent. As to integral (12) we notice that [2]

$$
\begin{aligned}
& \int_{0}^{\infty} x^{-2+a}([x]-x) \sin (b \log x) d x \leq \int_{0}^{\infty} \mid x^{-2+\mathrm{a}} \\
& ([x]-x) \sin (\operatorname{blog} x) \mid \mathrm{d} x \leq \int_{0}^{\infty} x^{-2+a}((x)) d x
\end{aligned}
$$

(where $((z))$ is the fractional part of $z, 0 \leq((z))<1$ )

$$
=\lim (\mathrm{t} \rightarrow 0) \int_{0}^{1-\mathrm{t}} x^{-1+a} d x+\lim (\mathrm{t} \rightarrow 0) \int_{1+\mathrm{t}}^{\infty} x^{-2+a}((x)) d x
$$

( $t$ is a very small positive number) (Since $((x))=x$ whenever $0 \leq x<1)$

$$
\begin{aligned}
& =\frac{1}{a}+\lim (t \rightarrow 0) \int_{1+t}^{\infty} x^{-2+a}((x)) d x \\
& <\frac{1}{a}+\lim (t \rightarrow 0) \int_{1+\mathrm{t}}^{\infty} x^{-2+a} d x=\frac{1}{a}+\frac{1}{a-1}
\end{aligned}
$$

${ }^{*}$ Corresponding author: Fayez Fok Al Adeh, President of the Syrian Cosmological Society, P.O.Box:13187, Damascus, Syria, Tel: 00963-11-2776729, 2713005 E-mail: hayfa@scs-net.org

Received June 09, 2016; Accepted December 13, 2016; Published December 27, 2016

Citation: Adeh FFA (2016) How to Prove the Riemann Hypothesis. J Generalized Lie Theory Appl 10: 250. doi:10.4172/1736-4337.1000250

Copyright: (c) 2016 Adeh FFA. This is an open-access article distributed under the terms of the Creative Commons Attribution License, which permits unrestricted use, distribution, and reproduction in any medium, provided the original author and source are credited. 
And as to integral (13) $\int_{0}^{\infty} y^{-1-a}([y]-y) \sin (b \log y) d y$

$\leq \int_{0}^{\infty}\left|y^{-1-a}([y]-y) \sin (b \log y)\right| d y$

$\leq \int_{0}^{\infty} y^{-1-a}((y)) d y$

$=\lim (t \rightarrow 0) \int_{0}^{1-\mathrm{t}} y^{-a} d y+\lim (t \rightarrow 0) \int_{1+\mathrm{t}}^{\infty} y^{-1-a}((y)) d y$

( $t$ is a very small positive number) (since $((y))=y$ whenever $0 \leq y<1)$

$$
\begin{aligned}
& =\frac{1}{1-a}+\lim (\mathrm{t} \rightarrow 0) \int_{1+\mathrm{t}}^{\infty} y^{-1-a}((y)) d y \\
& <\frac{1}{1-a}+\int_{1+t}^{\infty} y^{-1-a} d y=\frac{1}{1-a}+\frac{1}{a}
\end{aligned}
$$

Since the limits of integration do not involve $x$ or $y$, the product can be expressed as the double integral,

$\int_{0}^{\infty} \int_{0}^{\infty} x^{-2+a} y^{-1-a}([x]-x)([y]-y) \sin (b \log y) \sin (b \log x) d x d y=0$

Thus $\int_{0}^{\infty} \int_{0}^{\infty} x^{-2+a} y^{-1-a}([x]-x)([y]-y)$

$(\cos (b \log y+b \log x)-\cos (b \log y-b \log x)) d x d y=0$

$\int_{0}^{\infty} \int_{0}^{\infty} x^{-2+a} y^{-1-a}([x]-x)([y]-y)\left(\cos (b \log x y)-\cos \left(b \log \frac{y}{x}\right)\right) d x d y=0$

That is,

$\int_{0}^{\infty} \int_{0}^{\infty} x^{-2+a} y^{-1-a}([x]-x)([y]-y) \cos (b \log x y) d x d y=$

$\int_{0}^{\infty} \int_{0}^{\infty} x^{-2+a} y^{-1-a}([x]-x)([y]-y) \cos \left(b \log \frac{y}{x}\right) d x d y$

Consider the integral on the right-hand side of equation (17)

$\int_{0}^{\infty} \int_{0}^{\infty} x^{-2+a} y^{-1-a}([x]-x)([y]-y) \cos \left(b \log \frac{y}{x}\right) d x d y$

In this integral make the substitution $\mathrm{x}=\frac{1}{\mathrm{z}} \mathrm{dx}=\frac{-d z}{z^{2}}$

The integral becomes,

$\int_{0}^{\infty} \int_{\infty}^{0} z^{2-a} y^{-1-a}\left(\left[\frac{1}{z}\right]-\frac{1}{z}\right)([y]-y) \cos (b \log z y) \frac{-d z}{z^{2}} d y$

That is,

$-\int_{0}^{\infty} \int_{0}^{\infty} z^{-a} y^{-1-a}\left(\left[\frac{1}{z}\right]-\frac{1}{z}\right)([y]-y) \cos (b \log z y) d z d y$

This is equivalent to,

$\int_{0}^{\infty} \int_{0}^{\infty} z^{-a} y^{-1-a}\left(\left[\frac{1}{z}\right]-\frac{1}{z}\right)([y]-y) \cos (b \log z y) d z d y$

If we replace the dummy variable $z$ by the dummy variable $x$, the integral takes the form [3]

$\int_{0}^{\infty} \int_{0}^{\infty} x^{-a} y^{-1-a}\left(\left[\frac{1}{x}\right]-\frac{1}{x}\right)([y]-y) \cos (b \log x y) d x d y$

Rewrite this integral in the equivalent form,

$\int_{0}^{\infty} \int_{0}^{\infty} x^{-2+a} y^{-1-a}\left(x^{2-2 a}\left[\frac{1}{x}\right]-\frac{x^{2-2 a}}{x}\right)([y]-y) \cos (b \log x y) d x d y$
Thus equation 17 becomes,

$\int_{0}^{\infty} \int_{0}^{\infty} x^{-2+a} y^{-1-a}([x]-x)([y]-y) \cos (b \log x y) d x d y=$

$\int_{0}^{\infty} \int_{0}^{\infty} x^{-2+a} y^{-1-a}\left(x^{2-2 a}\left[\frac{1}{x}\right]-\frac{x^{2-2 a}}{x}\right)([y]-y) \cos (b \log x y) d x d y$

Write the last equation in the form,

$\int_{0}^{\infty} \int_{0}^{\infty} x^{-2+a} y^{-1-a}([y]-y) \cos (b \log x y)\left\{\left(x^{2-2 a}[-]-\frac{22}{x}\right)-([x]-x)\right\} d x$

$d y=0$

Let $p<0$ be an arbitrary small positive number. We consider the following regions in the $x-y$ plane [4].

The region of integration $I=[0, \infty) \times[0, \infty)$

The large region $I 1=[p, \infty) \times[p, \infty)$

The narrow strip $I 2=[p, \infty) \times[0, p]$

The narrow strip $I 3=[0, p] \times[0, \infty)$

Note that,

$I=I 1 \cup I 2 \cup I 3$

Denote the integer and in the left hand side of equation (25) by:

$$
\begin{aligned}
& F(x, y)=x^{-2+a} y^{-1-a}([y]-y) \cos (b \log x y) \\
& \left\{\left(\left(x^{2-2 a}\left[\frac{1}{x}\right]-\frac{x^{2-2 a}}{x}\right)-([x]-x)\right\}\right.
\end{aligned}
$$

Let us find the limit of $F(x, y)$ as $x \rightarrow \infty$ and $y \rightarrow \infty$. This limit is given by: $\lim x^{-a} y^{-1-a}[-((y))] \cos (\operatorname{blog} x y)\left[-\left(\left(\frac{1}{x}\right)\right)+((x)) x^{2 a-2}\right]$

$((\mathrm{z}))$ is the fractional part of the number $z, 0 \leq((z))<1$

The above limit vanishes, since all the functions $[-((y))], \cos (\operatorname{blog} x y),-\left(\left(\frac{1}{x}\right)\right)$, and $((x))$ remain bounded as $x \rightarrow \infty$ and $y \rightarrow \infty$

Note that the function $F(x, y)$ is defined and bounded in the region I 1 . We can prove that the integral,

$\iint_{I 1} F(x, y) d x d y$ is bounded as follows

$\leq \iiint_{I 1} x^{-a} y^{-1-a}[-((y))] \cos (\operatorname{blog} x y)\left[-\left(\left(\frac{1}{x}\right)\right)+\right.$

$\left.((x)) x^{2 a-2}\right] d x d y$

$\leq \iint_{I 1} x^{-a} y^{-1-a}[-((y))] \cos (\operatorname{blog} x y)\left[-\left(\left(\frac{1}{x}\right)\right)+\right.$

$\left.((x)) x^{2 a-2}\right] d x d y$

$=\mid \int_{p}^{\infty}\left(\int_{p}^{\infty} x^{-a} \cos (\operatorname{blog} x y)\left[-\left(\left(\frac{1}{x}\right)\right)+((x)) x^{2 a-2}\right] d x\right)$

$y^{-1-a}[-((y))] d y \mid$

$\leq \int_{p}^{\infty}\left|\left(\int_{p}^{\infty} x^{-a} \cos (\operatorname{blog} x y)\left[-\left(\left(\frac{1}{x}\right)\right)+((x)) x^{2 a-2}\right] d x\right)\right| y^{-1-a}[-((y))] \mid d y$

$\leq \int_{P}^{\infty}\left(\int_{P}^{\infty} x^{-a}|\cos (\operatorname{blog} x y)|\left|\left[-\left(\left(\frac{1}{x}\right)\right)+((x)) x^{2 a-2}\right]\right| d x\right)\left|y^{-1-a}[-((y))]\right| d y$ 
$<\int_{p}^{\infty} x^{-a}\left[\left(\left(\frac{1}{x}\right)\right)+((x)) x^{2 a-2}\right] d x \int_{p}^{\infty} y^{-1-a}$

$=\frac{1}{a p^{a}} \int_{p}^{\infty} x^{-a}\left[\left(\left(\frac{1}{x}\right)\right)+((x)) x^{2 a-2}\right] d x$

$=\frac{1}{a p^{a}}\left\{\lim (t \rightarrow 0) \int_{p}^{1-t} x^{-a}\left[\left(\left(\frac{1}{x}\right)\right)+((x)) x^{2 a-2}\right] d x+\lim (t \rightarrow 0) \int_{1+t}^{\infty} x^{-a}\left[\left(\left(\frac{1}{x}\right)\right)+((x)) x^{2 a-2}\right] d x\right\}$

Where $t$ is a very small arbitrary positive number. Since the integral: $\lim (t \rightarrow 0) \int_{P}^{1-t} x^{-a}\left[\left(\left(\frac{1}{x}\right)\right)+((x)) x^{2 a-2}\right] d x$ is bounded, it remains to show that $\lim (t \rightarrow 0)$

$\int_{1+t}^{\infty} x^{-a}\left[\left(\left(\frac{1}{x}\right)\right)+((x)) x^{2 a-2}\right] d x$ is bounded.

Since $x>1$, then $\left(\left(\frac{1}{x}\right)\right)=\frac{1}{x}$ and wehave $=\lim (t \rightarrow 0) \int_{1+t}^{\infty} x^{-a}\left[\frac{1}{x}+((x)) x^{2 a-2}\right] d x$

$=\lim (t \rightarrow 0) \int_{1+t}^{\infty} x^{-a}\left[\frac{1}{x}+((x)) x^{2 a-2}\right] d x$

$=\lim (t \rightarrow 0) \int_{1+t}^{\infty}\left[x^{-a-1}+((x)) x^{a-2}\right] d x$

$<\lim (t \rightarrow 0) \int_{1+t}^{\infty}\left[x^{-a-1}+x^{a-2}\right] d x$

$=\frac{1}{a(1-a)}$

Hence the boundedness of the integral $\iint_{I I} F(x, y) d x d y$ is proved. Consider the region:

$\mathrm{I} 4=\mathrm{I} 2 \cup \mathrm{I} 3$

We know that,

$0=\iint_{I} F(x, y) d x d y=\iint_{I 1} F(x, y) d x d y+\iint_{I 4} F(x, y) d x d y$ and that,

$\iint_{I 1} F(x, y) d x d y$ is bounded

From which we deduce that the integral [5]

$\iint_{I 4} F(x, y) d x d y$ is bounded

Remember that,

$\iint_{I 4} F(x, y) d x d y=\iint_{I 2} F(x, y) d x d y+\iint_{I 3} F(x, y) d x d y$

Consider the integral,

$\iint_{I 2} F(x, y) d x d y \leq\left|\iint_{I 2} F(x, y) d x d y\right|$

$\left|\int_{0}^{P}\left(\int_{P}^{\infty} x^{-a}\left\{\left(\left(\frac{1}{x}\right)\right)-((x)) x^{2 a-2}\right\} \cos (b \log x y) d x\right) \frac{1}{y^{a}} d \mathrm{y}\right|$

$\leq \int_{0}^{P}\left|\int_{P}^{\infty}\left(x^{-a}\left\{\left(\left(\frac{1}{x}\right)\right)-((x)) x^{2 a-2}\right\}|| \cos (b \log x y) d x\right)\right| \frac{1}{y^{a}} d \mathrm{y}$

$\leq \int_{0}^{P} \mid \int_{P}^{\infty}\left(x^{-a}\left\{\left(\left(\frac{1}{x}\right)\right)-((x)) x^{2 a-2}\right\}|| \cos (b \log x y) \mid d x\right) \frac{1}{y^{a}} d \mathrm{y}$

$$
\leq \int_{P}^{\infty}\left|x^{-a}\left\{\left(\left(\frac{1}{x}\right)\right)-((x)) x^{2 a-2}\right\}\right| d \mathrm{x} \times \int_{0}^{P} \frac{1}{y^{a}} d y
$$

(This is because in this region $((y))=y$ ). It is evident that the integral $\int_{P}^{\infty}\left|x^{-a}\left\{\left(\left(\frac{1}{x}\right)\right)-((x)) x^{2 a-2}\right\}\right| d x$ is bounded, this was proved in the course of proving that the integral $\iint_{\int} F(x, y) d x d y$ is bounded. Also it is evident that the integral,

$\int_{0}^{P} \frac{1}{y^{a}} d y$

is bounded. Thus we deduce that the integral (40) $\iint_{I 2} F(x, y) d x d y$ is bounded.

Hence, according to equation (39), the integral $\iint_{I 3} F(x, y) d x d y$ is bounded [6].

Now consider the integral,

$\iint_{I 3} F(x, y) d x d y$

We write it in the form:

$\iint_{I 3} F(x, y) d x d y=\int_{0}^{p}\left(\int_{0}^{\infty} y^{-1-a}((y)) \cos (b \log x y) d y\right) \frac{\left\{\left(\left(\frac{1}{x}\right)\right)-x^{2 a-1}\right\}}{x^{a}} d x$

(This is because in this region $((\mathrm{x}))=\mathrm{x}$ )

$\leq\left|\int_{0}^{p}\left(\int_{0}^{\infty} y^{-1-a}((y)) \cos (b \log x y) d y\right) \frac{\left\{\left(\left(\frac{1}{x}\right)\right)-x^{2 a-1}\right\}}{x^{\mathrm{a}}} d x\right|$

$\leq \int_{0}^{p}\left|\left(\int_{0}^{\infty} y^{-1-a}((y)) \cos (b \log x y) \mathrm{d} y\right)\right|\left|\frac{\left\{\left(\left(\frac{1}{x}\right)\right)-x^{2 a-1}\right\}}{x^{a}}\right| d x$

$\leq \int_{0}^{p}\left(\int_{0}^{\infty} y^{-1-a}((y)) d y\right)\left|\frac{\left\{\left(\left(\frac{1}{x}\right)\right)-x^{2 a-1}\right\}}{x^{a}}\right| d x$

Now we consider the integral with respect to $y$,

$\int_{0}^{\infty} y y^{-1-a}((y)) d y$

$=(\lim t \rightarrow 0) \int_{0}^{1-t} y^{-1-a} \times y d y+(\lim t \rightarrow 0) \int_{1+t}^{\infty} y^{-1-a}((y)) d y$

(where $t$ is a very small arbitrary positive number). (Note that $((y))=y$ whenever $0 \leq y<1)$.

Thus we have $(\lim t \rightarrow 0) \int_{1+t}^{\infty} y^{-1-a}((y)) d y<(\lim t \rightarrow 0) \int_{1+t}^{\infty} y^{-1-a} d y=\frac{1}{a}$

and $(\lim t \rightarrow 0) \int_{0}^{1-t} y^{-1-a} \times y d y=\frac{1}{1-a}$

Hence the integral (43) $\int_{0}^{\infty} y^{-1-a}((y)) d y$ is bounded.

Since $\left|\int_{0}^{\infty} y^{-1-a}((y)) \cos (b \log x y) d y\right| \leq \int_{0}^{\infty} y^{-1-a}((y)) d y$, we conclude that the integral $\left|\int_{0}^{\infty} y^{-1-a}((y)) \cos (b \log x y) d y\right| \quad$ is a bounded function of $x$. Let this function be $H(x)$. Thus we have,

$$
\left|\int_{0}^{\infty} y^{-1-a}((y)) \cos (b \log x y) d y\right|=H(x) \leq K(K \text { is a positive }
$$

number) 
Now equation (44) gives us,

$$
-K \leq \int_{0}^{\infty} y^{-1-a}((y)) \cos (b \log x y) d y \leq K
$$

According to equation (42) we have,

$\iint_{I 3} F(x, y) d x d y=\int_{0}^{p}\left(\int_{0}^{\infty} y^{-1-a}((y)) \cos (b \log x y) d y\right) \frac{\left\{\left(\left(\frac{1}{x}\right)\right)-x^{2 a-1}\right\}}{x^{a}} d x$

$\geq \int_{0}^{p}(-K) \frac{\left\{\left(\left(\frac{1}{x}\right)\right)-x^{2 a-1}\right\}}{x^{a}} d x=K \int_{p}^{0} \frac{\left\{\left(\left(\frac{1}{x}\right)\right)-x^{2 a-1}\right\}}{x^{a}} d x$

Since $\iint_{I 3} F(x, y) d x d y$ is bounded, then $\int_{p}^{0} \frac{\left\{\left(\left(\frac{1}{x}\right)\right)-x^{2 a-1}\right\}}{x^{a}} \mathrm{dx}$ is also bounded. Therefore the integral,

$G=\int_{0}^{p} \frac{\left\{\left(\left(\frac{1}{x}\right)\right)-x^{2 a-1}\right\}}{x^{a}} d x$ is bounded

We denote the integer and of (47) by:

$$
F=\frac{1}{x^{a}}\left\{\left(\left(\frac{1}{x}\right)\right)-x^{2 a-1}\right\}
$$

Let $\delta G[F]$ be the variation of the integral $G$ due to the variation of the integrand $\delta F$.

Since,

$G[F]=\int F d x$ (the integral (49) is indefinite)

(here we do not consider a as a parameter, rather we consider it as a given exponent)

We deduce that $\frac{\delta G[F]}{\delta F(x)}=1$

that is,

$\delta G[F]=\delta F(x)$

But we have,

$\delta G[F]=\int d x \frac{\delta G[F]}{\delta F(x)} \delta F(x)$ (the integral (51) is indefinite)

Using equation (50) we deduce that,

$\delta G[F]=\int d x F(x)$ ( the integral (52) is indefinite)

Since $G[F]$ is bounded across the elementary interval $[0, p]$, we must have that,

$\delta G[F]$ is bounded across this interval

From (52) we conclude that,

$$
\delta G=\int_{0}^{p} d x \delta F(x)=\int_{0}^{p} d x \frac{d F}{d x} \delta x=[F \delta x](\text { at } x=p)-[F \delta x](\text { at } x=0)
$$

Since the value of $[F \delta x]$ (at $x=p$ )is bounded, we deduce from equation (54) that,

$\lim (x \rightarrow 0) F \delta x$ must remain bounded.

Thus we must have that

$(\lim x \rightarrow 0)\left[\delta x \frac{1}{x^{a}}\left\{\left(\left(\frac{1}{x}\right)\right)-x^{2 a-1}\right\}\right]$ is bounded.

First we compute,

$(\lim x \rightarrow 0) \frac{\delta x}{x^{a}}$

Applying L 'Hospital ' rule we get, $(\lim x \rightarrow 0) \frac{\delta x}{x^{a}}=(\lim x \rightarrow 0) \frac{1}{a} \times x^{1-a} \times \frac{d(\delta x)}{d x}=0$

We conclude from (56) that the product,

$0 \times(\lim x \rightarrow 0)\left\{\left(\left(\frac{1}{x}\right)\right)-x^{2 a-1}\right\}$ must remain bounded.

Assume that $a=0.5$. (Remember that we considered a as a given exponent)This value $\mathrm{a}=0.5$ will guarantee that the quantity $\left\{\left(\left(\frac{1}{x}\right)\right)-x^{2 a-1}\right\}$

will remain bounded in the limit as $(x \rightarrow 0)$. Therefore, in this case $(\mathrm{a}=0.5)$ (56) will approach zero as $(\mathrm{x} \rightarrow 0)$ and hence remain bounded.

Now suppose that $\mathrm{a}<0.5$. In this case we consider a as a parameter. Hence we have,

$G_{a}[x]=\int d x \frac{F(x, a)}{x} x$ (the integral (60) is indefinite )

Thus,

$\frac{\delta G_{a}[x]}{\delta x}=\frac{F(x, a)}{x}$

But we have that,

$\delta G_{a}[x]=\int d x \frac{\delta G_{a}[x]}{\delta x} \delta x$ (the integral (62) is indefinite)

Substituting from (61) we get,

$\delta G_{a}[x]=\int d x \frac{F(x, a)}{x} \delta x$ (the integral (63) is indefinite)

We return to equation (49) and write,

$G=\lim (t \rightarrow 0) \int_{t}^{p} F d x(t$ is a very small positive number $0<t<p)(64)$

$=\{F x($ at $p)-\lim (t \rightarrow 0) F x($ at $t)\}-\lim (t \rightarrow 0) \int_{t}^{p} x d F$

Let us compute,

$\lim (t \rightarrow 0) F x($ at $t)=\lim (t \rightarrow 0) t^{1-a}\left(\left(\frac{1}{t}\right)\right)-t^{a}=0$

Thus equation (64) reduces to,

$G-F x($ at $p)=-\lim (t \rightarrow 0) \int_{\mathrm{t}}^{p} x d F$

Note that the left - hand side of equation (66) is bounded. Equation (63) gives us,

$\delta G_{a}=\lim (t \rightarrow 0) \int_{\mathrm{t}}^{p} d x \frac{F}{x} \delta x$

( $t$ is the same small positive number $0<t<p$ )

We can easily prove that the two integrals $\int_{t}^{p} x d F$ and $\int_{t}^{p} d x \frac{F}{x} \delta x$ are absolutely convergent. Since the limits of integration do not involve any variable, we form the product of (66) and (67)

$K=\lim (t \rightarrow 0) \int_{t}^{p} \int_{t}^{p} x d F \times d x \frac{F}{x} \delta x=\lim (t \rightarrow 0) \int_{\mathrm{t}}^{p} F d F \times \int_{t}^{p} \delta x d x$

( $K$ is a bounded quantity)

That is,

$K=\lim (t \rightarrow 0)\left[\frac{F^{2}}{2}(\right.$ at $p)-\frac{F^{2}}{2}($ at $\left.t)\right] \times[\delta x($ at $p)-\delta x($ at $t)]$

We conclude from this equation that, 
$\left\{\left[\frac{F^{2}}{2}(\right.\right.$ at $p)-\lim (t \rightarrow 0) \frac{F^{2}}{2}($ at $\left.t)\right] \times[\delta x($ at $\left.p)]\right\}$ is bounded. (70)

(Since $\lim (x \rightarrow 0) \delta x=0$, which is the same thing as $\lim (t \rightarrow 0) \delta x=0$ ) Since $\frac{F^{2}}{2}($ at $p)$ is bounded, we deduce at once that $\frac{F^{2}}{2}$ must remain bounded in the limit as $(t \rightarrow 0)$, which is the same thing as saying that $F$ must remain bounded in the limit as $(x \rightarrow 0)$. Therefore,

$\lim (x \rightarrow 0) \frac{\left(\left(\frac{1}{x}\right)\right)-x^{2 a-1}}{x^{a}}$ must remain bounded

But,

$\lim (x \rightarrow 0) \frac{\left(\left(\frac{1}{x}\right)\right)-x^{2 a-1}}{x^{a}} \lim (x \rightarrow 0) \frac{x^{1-2 a}}{x^{1-2 a}} \times \frac{\left(\left(\frac{1}{x}\right)\right)-x^{2 a-1}}{x^{a}}$

$\lim (x \rightarrow 0) \frac{x^{1-2 a}\left(\left(\frac{1}{x}\right)\right)-1}{x^{1-a}}=\lim (x \rightarrow 0) \frac{-1}{x^{1-a}}$

It is evident that this last limit is unbounded. This contradicts our conclusion (71) that:

$\lim (x \rightarrow 0) \frac{\left(\left(\frac{1}{x}\right)\right)-x^{2 a-1}}{x^{a}}$ must remain bounded (for $a<0.5$ )

Therefore the case $a<0.5$ is rejected. We verify here that, for $a=0.5$ (71) remains bounded as $(x \rightarrow 0)$.

We have that:

$\left(\left(\frac{1}{x}\right)\right)-x^{2 a-1}<1-x^{2 a-1}$

Therefore:

$\lim (a \rightarrow 0.5)(x \rightarrow 0) \frac{\left(\left(\frac{1}{x}\right)\right)-x^{2 a-1}}{x^{a}}<\lim (a \rightarrow 0.5)(x \rightarrow 0) \frac{1-x^{2 a-1}}{x^{a}}$

We consider the limit:

$\lim (a \rightarrow 0.5)(x \rightarrow 0) \frac{1-x^{2 a-1}}{x^{a}}$

We write:

$a=(\lim x \rightarrow 0)(0.5+x)$

Hence we get:

$\lim (a \rightarrow 0.5)(x \rightarrow 0) \mathrm{x}^{2 a-1}=\lim (x \rightarrow 0) x^{2(0.5+x)-1}=\lim (x \rightarrow 0) x^{2 x}=1$

(Since $\lim (x \rightarrow 0) x^{x}=1$ )

Therefore we must apply L 'Hospital ' rule with respect to $x$ in the limiting process (75)

$\lim (a \rightarrow 0.5)(x \rightarrow 0) \frac{1-x^{2 a-1}}{x^{a}}=\lim (a \rightarrow 0.5)(x \rightarrow 0) \frac{-(2 a-1) x^{2 a-2}}{a x^{a-1}}$

$\lim (a \rightarrow 0.5)(x \rightarrow 0) \frac{\left(\frac{1}{a}-2\right)}{x^{1-a}}$

Now we write again,

$a=(\lim x \rightarrow 0)(0.5+x)$

Citation: Adeh FFA (2016) How to Prove the Riemann Hypothesis. J Generalized Lie Theory Appl 10: 250. doi:10.4172/1736-4337.1000250
Thus the limit (78) becomes:

$\lim (a \rightarrow 0.5)(x \rightarrow 0) \frac{\left(\frac{1}{a}-2\right)}{\frac{15 a}{x^{-a}}}=\lim (x \rightarrow 0) \frac{(0.5+x)^{-1}-2}{x^{-0.5 x}}=\lim (x \rightarrow 0) \frac{(0.5+x)^{-1}-2}{x^{0.5} \times x^{-x}}$
$=\lim (x \rightarrow 0) \frac{\left(\frac{\left.5^{-x}+x\right)^{-1}-2}{x^{0.5}}\left(\text { Since } \lim (x \rightarrow 0) x^{-x}=1\right)\right.}{}$

We must apply L 'Hospital ' rule,

$$
\lim (x \rightarrow 0) \frac{(0.5+x)^{-1}-2}{x^{0.5}}=\lim (x \rightarrow 0) \frac{-(0.5+x)^{-2}}{0.5 x^{0.5}}=\lim (x \rightarrow 0) \frac{-2 \times x^{0.5}}{(0.5+x)^{2}}=0
$$

Thus we have verified here that, for $\mathrm{a}=0.5$ (71) approaches zero as $(x \rightarrow 0)$ and hence remains bounded.

We consider the case $a>0.5$. This case is also rejected, since according to the functional equation, if $(\zeta(S)=0)(s=a+b i)$ has a root with $a>0.5$, then it must have another root with another value of $a<0.5$. But we have already rejected this last case with $a<0.5$

Thus we are left with the only possible value of a which is $a=0.5$

Therefore $a=0.5$

This proves the Riemann Hypothesis.

\section{Conclusion}

The Riemann Hypothesis is now proved. The hypothesis states that the nontrivial zeros of the Riemann zeta function have real part equal to 0.5 . I assume that any such zero is $s=a+b i$. I use integral calculus in the first part of the proof. In the second part I employ variational calculus. Through equations (50) to (59) I consider (a) as a fixed exponent, and verify that $a=0.5$. From equation (60) onward I view (a) as a parameter $(a<0.5)$ and arrive at a contradiction. At the end of the proof (from equation (73)) and through the assumption that (a) is a parameter, I verify again that $a=0.5$

\section{References}

1. Titch marsh EC (1999) The Theory of the Riemann zeta - function. London: Oxford University Press.

2. Apostol Tom M (1974) Mathematical Analysis. Reading, Massachusetts Addison - wesley Publishing Company.

3. Edwards HM (1974) Riemann's zeta function. New York: Academic Press, Inc.

4. Apostol Tom M (1976) Introduction to Analytic Number Theory. New York Springer - Verlag.

5. Neal K (1984) P-adic Numbers, P-adic Analysis, and Zeta-Functions. New York: Springer - Verlag.

6. Walter G, Joachim R (1996) Field Quantization. Berlin: Springer - Verlag.

OMICS International: Open Access Publication Benefits \& Features

Unique features:

- Increased global visibility of articles through worldwide distribution and indexing

- Showcasing recent research output in a timely and updated manner

- Special issues on the current trends of scientific research

Special features:

- 700+ Open Access Journals

$50,000+$ editorial tean

Rapid review process

Quality and quick editorial, review and publication processing

Indexing at major indexing services

Sharing Option: Social Networking Enabled

Authors, Reviewers and Editors rewarded with online Scientific Credits

Better discount for your subsequent articles

Submit your manuscript at: http://www.omicsonline.org/submission 